\title{
Valproic acid upregulates NKG2D ligand expression and enhances susceptibility of human renal carcinoma cells to NK cell-mediated cytotoxicity
}

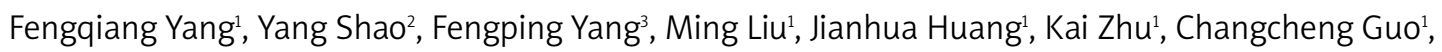
Jun Luo', Wei Li', Bin Yang', Jumei Shi', Junhua Zheng ${ }^{1}$

1Department of Urology, Tenth People's Hospital of Tongji University, Shanghai, China 2Department of Urology, Shanghai Armed Police Hospital, Shanghai, China

3Department of Medicine, People's Hospital in Xinyuan County Xinjiang Province, Xinjiang, China

${ }^{4}$ Department of Hematology, Shanghai Tenth People's Hospital, Tongji University School of Medicine, Shanghai, China

Submitted: 13 September 2012

Accepted: 31 October 2012

Arch Med Sci 2013; 9, 2: 323-331

DOI: 10.5114/aoms.2013.34413

Copyright $\odot 2013$ Termedia \& Banach

\begin{abstract}
Introduction: We aimed to investigate the effect of valproic acid (VPA) on NKG2D ligand expression in human renal carcinoma cell lines and to investigate the mechanisms.

Material and methods: Different concentrations of VPA from $0.5 \mathrm{mM}$ to $8.0 \mathrm{mM}$ were applied to 786-O and ACHN cell lines, respectively. Cell viability after treatment with VPA was determined by flow cytometry (FCM). Real-time PCR and FCM were used to detect the changes of $M R N A$ and protein level of NKG2D ligands (MICA/B and ULBPs) in the two cell lines treated with $4 \mathrm{mM} \mathrm{VPA.} \mathrm{The} \mathrm{cyto-}$ toxicity assay and CD107a mobilization assay were carried out to detect the cytotoxicity changes of NK cells against renal carcinoma cell lines after the same treatment.

Results: Valproic acid can efficiently upregulate MICA/B, ULBP1 and ULBP2 expression in the renal carcinoma cell lines at the mRNA and protein level $(p<0.05) .786-0$ and ACHN cells treated with VPA were more susceptible to killing by NK cells than untreated cells and the enhanced cytotoxicity of NK cells was blocked by the pretreatment of NK cells with anti-NKG2D monoclonal antibodies $(p<0.05)$.

Conclusions: Valproic acid can clearly induce the expression of NKG2D ligands of renal carcinoma cell lines, thereby enhancing the cytotoxicity of NK cells against renal carcinoma cell lines.
\end{abstract}

Key words: valproic acid, renal carcinoma cell, NKG2D ligands, NK cells.

\section{Introduction}

Renal cell carcinoma (RCC), which refers to cortically derived tumors of the renal parenchyma and encompasses a heterogeneous group of cancers, is the third most common urological cancer after prostate and bladder cancer [1]. Renal cell carcinoma is diagnosed in more than 120,000 patients in Europe and the USA every year and causes about 60,000 deaths annually. Of these patients, approximately one third, who have initially localized disease, relapse after nephrectomy, while 30\% present with metastatic RCC at diagnosis [2]. The overall poor prognosis of RCC is part-

\author{
Corresponding author: \\ Junhua Zheng MD, PhD \\ Department of Urology \\ Tenth People's Hospital \\ of Tongji University \\ Shanghai 200072, China \\ Phone: +86 02166300588 \\ E-mail: \\ zhengjh0471@sina.com
}


ly because of the particular resistance to conventional chemotherapy and radiotherapy in most cases [3]. Thus, alternative treatment strategies that focus on the development of immunotherapy are highly need.

Natural killer (NK) cells are large granular cells of the immune system that constitute the third major lymphocyte subset and represent approximately $10-15 \%$ of circulating lymphocytes in blood [4]. Natural killer cells express a large number of different cell surface receptors and contribute to the elimination of virus-infected cells as well as to the antitumor immune response [4]. NKG2D is an activating receptor of NK cells which is thought to play an important role in tumor immune surveillance [5]. In recent years, several highly diversified MHC class I related molecules which have been shown to bind to the homodimeric C-type lectinlike NKG2D receptor have been found to be expressed in cytotoxic lymphocytes [6]. The human NKG2D ligands (NKG2DL) include the stressinducible surface glycoproteins MICA and MICB, expressed in many epithelial tumors and upon infection [7, 8], as well as the UL16 binding proteins (ULBPs), a multigene family with at least six functional members [9]. NKG2DL expression is known to potently stimulate antitumor responses in mice, which are critically dependent on NKG2DL expression levels on the tumor cell surface [10].

Valproic acid (VPA), a histone deacetylase inhibitor (HDAC-I), is a potent inducer of NKG2DLs such as MICA/B and ULBPs on tumor cells to enhance cytotoxicity of NK cells and host immune surveillance against tumor cells [11-13]. In the last years, VPA has shown potent antitumor effects in a variety of in vitro and in vivo systems [14] and has emerged as a promising drug for cancer treatment with well tolerable side effects [15]. However, the functional consequences of VPA treatment for cellular immunity in renal carcinoma cells remain unclear.

In our study, we investigated the effect of VPA on expression of NKG2D ligands in human renal carcinoma cell lines. In addition, we detected the mRNA and protein levels of NKG2D ligands in renal carcinoma cell lines treated with 4 mM VPA using real-time $P C R$ and flow cytometry. Furthermore, a cytotoxicity assay and CD107a mobilization assay were carried out to study the susceptibility of human renal carcinoma cells to NK cell-mediated cytotoxicity.

\section{Material and methods}

\section{Reagents and monoclonal antibodies}

Sodium valproate (VPA) and calcein-AM were obtained from Sigma (USA). Monoclonal antibodies (mAbs) specific to ULBP-1, ULBP-2, ULBP-3 and
anti-NKG2D were purchased from R\&D Systems (Minneapolis, MN, USA). PE-conjugated goat antimouse lgG, antimouse lgG1 Ab, goat anti-mouse PE Ab, anti-MICA/B-PE, anti-HLA-A/B,/C-FITC, antiCD56-PE and anti-CD107a-APC were all purchased from BD Pharmingen (San Diego, CA, USA). Annexin V-FITC and propidium iodide were purchased from eBioscience (USA).

\section{Cells}

Human renal carcinoma cell lines of 786-O and ACHN, as well as NK-92 cells, were purchased from American Type Culture Collection (ATCC, Manasas, VA, USA). 786-O and ACHN were cultured in RPMI-1640 supplemented with $100 \mathrm{U} / \mathrm{ml}$ penicillin, $100 \mu \mathrm{g} / \mathrm{ml}$ streptomycin, and 10\% fetal bovine serum (FBS). NK-92 cells were cultured in Minimum Essential alpha Medium containing $0.2 \mathrm{mM}$ inositol, $0.1 \mathrm{mM}$ 2-mercaptoethanol, $0.02 \mathrm{mM}$ folic acid, $17 \mathrm{mM}$ sodium bicarbonate, $12.5 \%$ horse serum, $12.5 \% \mathrm{FBS}$ and $100 \mathrm{U} / \mathrm{ml}$ recombinant human interleukin 2. All cells were cultured in a humidified atmosphere of $5 \% \mathrm{CO}_{2}$ in air at $37^{\circ} \mathrm{C}$.

\section{Immunofluorescence staining and flow cytometry}

786-O and ACHN cells were cultured in six-well tissue culture plates for $48 \mathrm{~h}$ at a concentration of $3 \times 10^{5}$ cells $/ \mathrm{ml}$, with different concentrations of VPA from $0.5 \mathrm{mM}$ to $0.8 \mathrm{mM}$. The expression of the NKG2D ligands and HLA-A/B/C on renal carcinoma cell lines was analyzed by immunofluorescence staining using anti-ULBP-1, anti-ULBP-2 and antiULBP-3, followed by secondary goat anti-mouse PE Ab, anti-MICA/B-PE and anti-HLA-A/B/C-FITC. In all experiments, cells were stained with propidium iodide $(1 \mu \mathrm{g} / \mu \mathrm{l})$ to assess cell viability. Aspecific fluorescence was assessed by using an isotypematched irrelevant mAb (R\&D Systems, Minneapolis, MN, USA) followed by the same secondary $\mathrm{mAb}$. Fluorescence was analyzed using a FACSCalibur flow cytometer (BD Biosciences, Franklin Lakes, NJ, USA). Data acquisition and flow cytometric analysis were carried out on a BD FACSCalibur using the CellQuest software package (BD Biosciences, Franklin Lakes, NJ, USA).

\section{RNA isolation and real-time PCR}

Total RNA from renal carcinoma cell lines, which had been treated with VPA and cultured for $24 \mathrm{~h}$, was extracted using TRIZOL [16] (Invitrogen, Carlsbad, CA, USA) according to the manufacturer's instructions. The concentration and quality of the extracted total RNA were determined by measuring OD260 and the OD260/OD280 ratio. The reverse transcription of $1 \mu \mathrm{g}$ RNA into cDNA was carried out using Superscript II reverse transcriptase (Takara, 
Table I. Primer sequences for real-time PCR

\begin{tabular}{|c|c|c|}
\hline Gene & Primer sequences & Product size [bp] \\
\hline MICA & $\begin{array}{c}\text { F:5'-CAGCTGCAAACGCCTCATATC-3' } \\
\text { R:5'-TGACCTATGAAACAGAGAAAATAAAAGC-3' }\end{array}$ & 87 \\
\hline MICB & $\begin{array}{l}\text { F: 5'-AGGAGAGGAGC AGAGGTTC AC-3' } \\
\text { R: 5'-TGGC ATAGC AGC AGAAAC ATA-3' }\end{array}$ & 91 \\
\hline ULBP1 & $\begin{array}{l}\text { F: 5'- AAAC AACAGAAAGTGGAC AGC A-3' } \\
\text { R: 5'- TACAATCCCCCAGTGAAATCTT-3' }\end{array}$ & 113 \\
\hline ULBP2 & $\begin{array}{l}\text { F: 5'- GAAAAGTGGGAGAATGAC AAGG-3' } \\
\text { R: 5'- TCAAGAAGTCCTC AAGCCATC-3' }\end{array}$ & 88 \\
\hline ULBP3 & $\begin{array}{l}\text { F: 5'- CTGGGGAAAAC AACTGGAAAT-3' } \\
\text { R: 5'- GCTTC ACACTC AC AAGAC ATCC-3' }\end{array}$ & 135 \\
\hline GAPDH & $\begin{array}{c}\text { F: 5'-TGCACC ACCAACTGCTTAGC-3' } \\
\text { R: 5'-GGC ATGGACTGTGGTC ATGAG-3' }\end{array}$ & 142 \\
\hline
\end{tabular}

Japan) and stored at $-80^{\circ} \mathrm{C}$ until use. Real-time PCR was performed using the $\mathrm{ABI}$ Prism 7900 sequence detection system (Applied Biosystems, USA). The average of the threshold cycles was used to interpolate standard curves and to calculate the transcript amount in samples using SDS version 2.1 software (Applied Biosystems, USA). Relative mRNA amount, normalized with glyceraldehyde 3-phosphate dehydrogenase (GAPDH), was expressed as arbitrary units and referred to untreated cells considered as calibrator. Real-time PCR was carried out using standard conditions, as previously described [17]. The primers used in real-time PCR are shown in Table I.

\section{Cytotoxicity assay}

NK cell cytotoxicity against renal carcinoma cell lines was assessed using the calcein-AM release assay, as described previously [18]. Renal carcinoma target cells $(T)$ were cultured in medium with or without $4 \mathrm{mM}$ VPA for $48 \mathrm{~h}$. Then, the NK cell cytotoxicity assay was carried out by incubating these cells with NK-92 effector cells (E) at various T/E ratios (1/20-1/5) for $3 \mathrm{~h}$ at $37^{\circ} \mathrm{C}$. In blocking experiments, anti-NKG2D mAbs were added to the NK cell suspension at $10 \mu \mathrm{g} / \mathrm{ml}$ and incubated at $37^{\circ} \mathrm{C}$ for $30 \mathrm{~min}$ before the addition of target cells. The number of dead cells was estimated by measuring the fluorescence intensity in medium. The fluorescence intensity in medium released spontaneously from target cells and the total maximum fluorescence intensity released from all target cells by treatment with $1 \%$ Triton X-100 were also determined. The percentage of target cells killed by NK-92 cells was calculated as follows: $100 x$ (sample protease activity - spontaneously released protease activity)/(total maximum protease activity spontaneously released protease activity).

\section{CD107a mobilization assay}

VPA-treated renal carcinoma cell lines were incubated with NK-92 cells at an E/T ratio of $2.5: 1$, and
NK cell-mediated cytotoxicity was evaluated using the lysosomal marker. Two microliters of APCCD107a mAb were added to the suspension of effector and target cell mixtures in 96-well round microplates and incubated for $3 \mathrm{~h}$ at $37^{\circ} \mathrm{C}$. One microliter of $2 \mathrm{mM}$ monensin (Sigma) in 100\% ethanol was included in the cell suspension to prevent the acidification of the endosomal compartment, which could alter the fluorescence of internalized CD107a: APC-CD107a mAb complexes. After incubation, the plate was centrifuged to the pellet cells and the supernatant was removed. Cell-cell conjugate was disrupted by washing the cell with PBS supplemented with $0.02 \%$ azide and $0.5 \mathrm{mM}$ EDTA. Samples were then stained with anti-CD3FITC and anti-CD56-PE to gate the CD3-CD56+ NK cell population, followed by flow cytometric analysis [12].

\section{Statistical analysis}

Data are presented as mean \pm SDs. Differences in the expression levels of NKG2D ligands in renal carcinoma cell lines were assessed using Student's $t$-test [19]. A value of $p$ less than 0.05 was considered statistically significant.

\section{Results}

VPA upregulates the expression of MICA/B, ULBP-1, and ULBP-2 on the surface of human renal carcinoma cell lines

To investigate the effect of VPA on the surface expression of NKG2D ligands in renal carcinoma cells, we initially performed a flow cytometric analysis on 786-O and ACHN cells after treatment with VPA. As shown in Figure $1 \mathrm{~A}$ and $1 \mathrm{~B}, 786-\mathrm{O}$ and $A C H N$ cells showed apparent upregulation of MICA/B, ULBP1, and ULBP2 after treated with VPA. However, the expression levels of HLA-A/B/C were not significantly influenced by VPA (Figure $1 \mathrm{C}$ ). The propidium iodide staining experiment showed that 
A
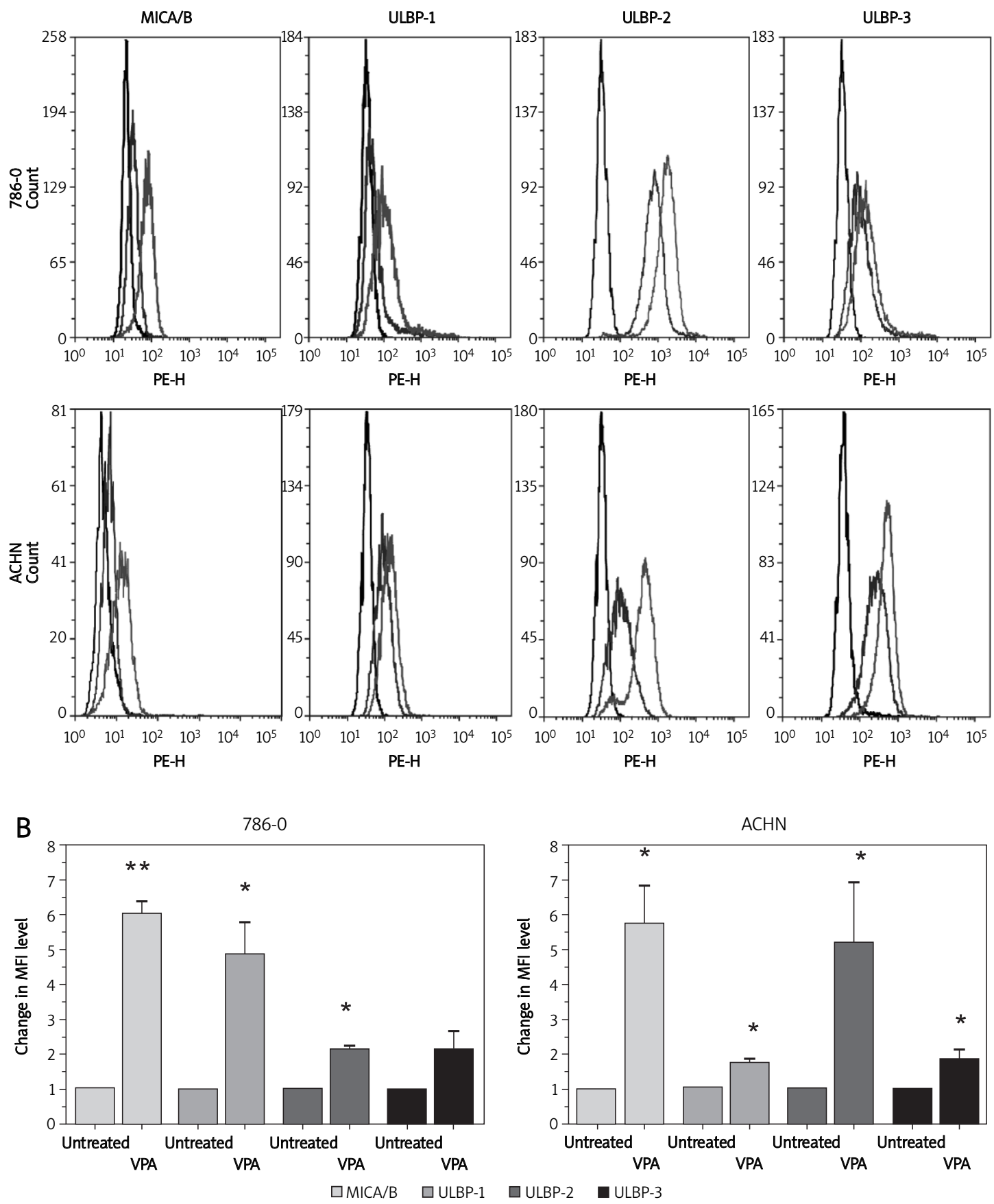

Figure 1. Up-regulation of NKG2D ligand (NKG2DL) expression on renal carcinoma cell lines by treatment with valproic acid (VPA). A - Histograms of NKG2DL expression in two renal carcinoma cell lines: purple lines represent untreated cells, black lines represent isotype control, green line represents VPA treated cells. B - Changes in the mean fluorescence intensity (MFI) level of MICA/B, ULBP-1, ULBP-2, ULBP-3 in the two renal carcinoma cell lines. The differences in the MFI levels are indicated as the mean $\pm S D$ from three independent experiments; ${ }^{*} p<0.05,{ }^{* *} p<0.01$

cell viability was not significantly changed by VPA from $0 \mathrm{mM}$ to $4 \mathrm{mM}$. However, cell apoptosis was significantly increased by VPA at $8 \mathrm{mM}$ (Figure 1 D). Therefore, we chose $4 \mathrm{mM}$ VPA for further analysis.

From the result of FCM analysis, we also found that the up-regulation of expression levels of ULBP-3 in the 786-O cell line was not of statistical significance
(Figures $1 \mathrm{~A}$ and $1 \mathrm{~B}$ ). That is, this ligand may not be induced by VPA in the 786-O cell line. Thus, we suggested that VPA could enhance the expression levels of most of the NKG2D ligands in 786-O and ACHN renal carcinoma cells.

We hypothesized that this influence was the consequence of up-regulation of their mRNA expression. To test this hypothesis, we isolated the 

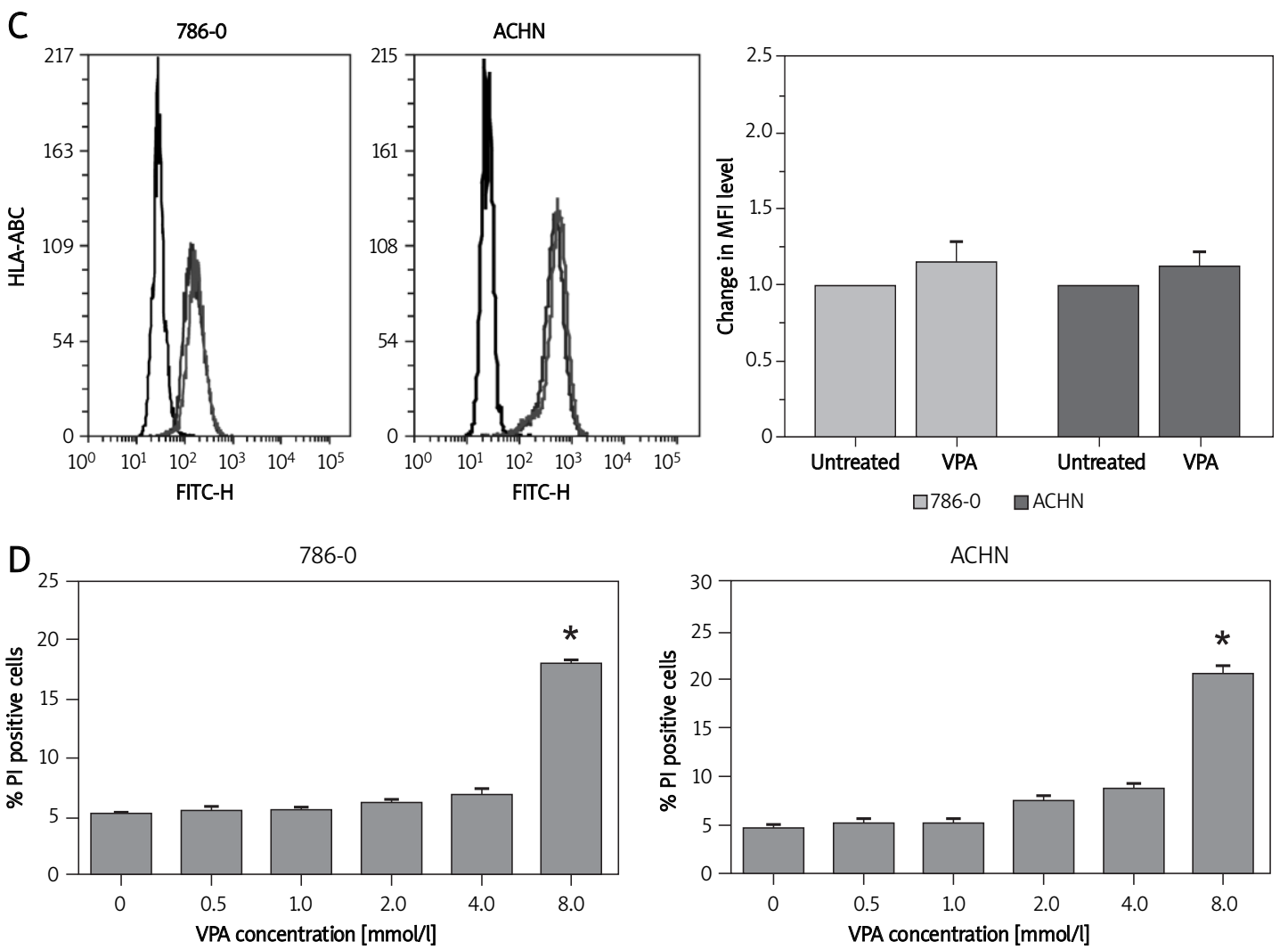

Figure 1. C - HLA-A/B/C expression in two renal carcinoma cell lines: purple lines represent untreated cells, black lines represent isotype control, green lines represent VPA treated cells. D-786-O and ACHN cell viability after treatment with different concentrations of VPA. The percent of propidium iodide (P/I)-positive cells was used to show the presence of different concentrations (micromolar) of VPA. Each column represents the difference of MFI level calculated by subtracting the MFI level of untreated cells from that of VPA treated cells

The differences in the MFI levels are indicated as the mean \pm SD from three independent experiments; ${ }^{*} p<0.05$

total RNA from 786-O and ACHN cells exposed to VPA inhibitors for $24 \mathrm{~h}$ and analyzed their mRNA levels by real-time PCR. We found that VPA in-

A

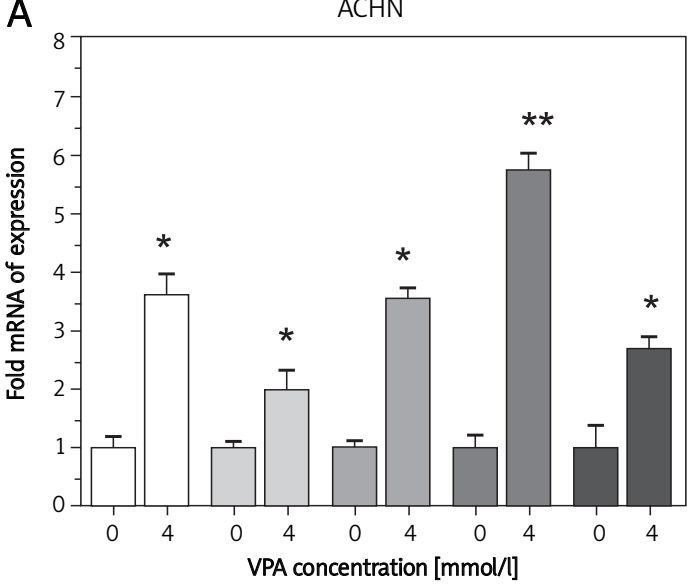

creased the mRNA levels of MICA, MICB, ULBP-1, and ULBP-2 in 786-O cells and the mRNA levels of MICA, MICB, ULBP-1, ULBP-2, and ULBP-3 in ACHN

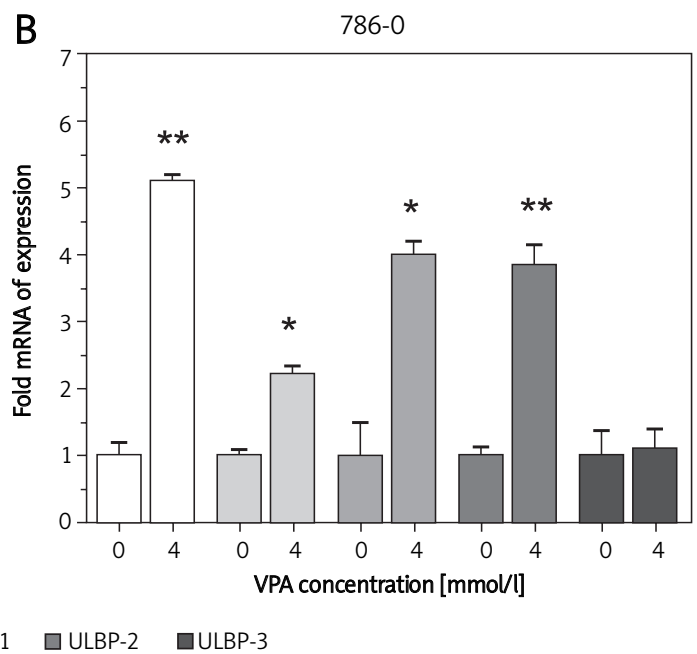

Figure 2. VPA increased the mRNA levels of NKG2D ligands (NKG2DL) in renal carcinoma cell lines. Real-time PCR analysis of total mRNA obtained from ACHN (A) or 786-O cells (B), untreated or treated with $4 \mathrm{mM}$ VPA for $24 \mathrm{~h}$. The relative amount of NKG2DL mRNA to GAPDH mRNA (NKG2DL/GAPDH) was used to represent expression levels of the NKG2DL gene

Each column and error bar represents the mean $\pm S D$ from three independent experiments; ${ }^{*} p<0.05,{ }^{* *} p<0.01$ 
cells (Figure 2). This result was consistent with the FCM analysis.

These results showed that VPA was able to enhance NKG2D ligand expression in renal carcinoma cell lines at both the mRNA and protein level.

\section{VPA enhances susceptibility of human renal carcinoma cells to NK cell-mediated cytotoxicity}

Two renal carcinoma cell lines were cultured for $48 \mathrm{~h}$ in the presence of $4.0 \mathrm{mM}$ VPA and the susceptibility to cytotoxicity of NK-92 cells was examined. As shown in Figure 3 A, VPA treatment of renal carcinoma cells increased the susceptibility to NK-92 cells. NK-92 cells pretreated with NKG2Dblocking antibody completely prevented the cytotoxic activity of NK-92 cells against all target cells treated with $4.0 \mathrm{mM}$ VPA (Figure $3 \mathrm{~B}$ ).

\section{VPA enhances NK cell degranulation against renal carcinoma cells}

Because changes in the expression of NKG2D ligands, such as MICA, MICB, ULBP-1 and ULBP-2, on tumor cells can modify the recognition and

B

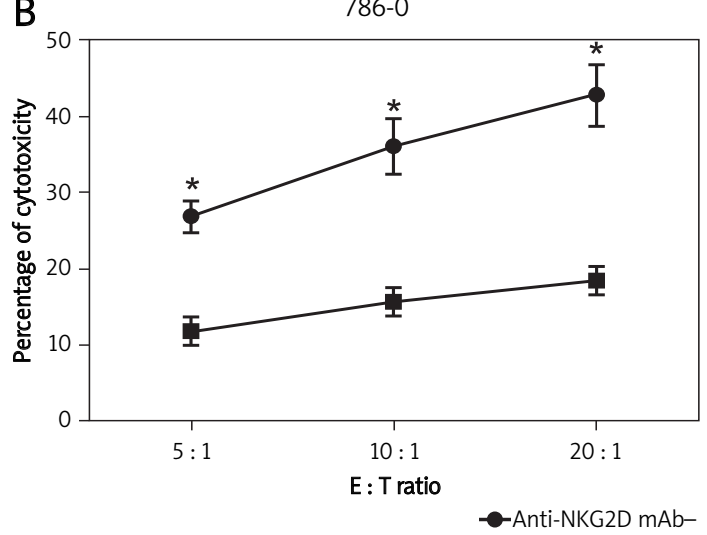

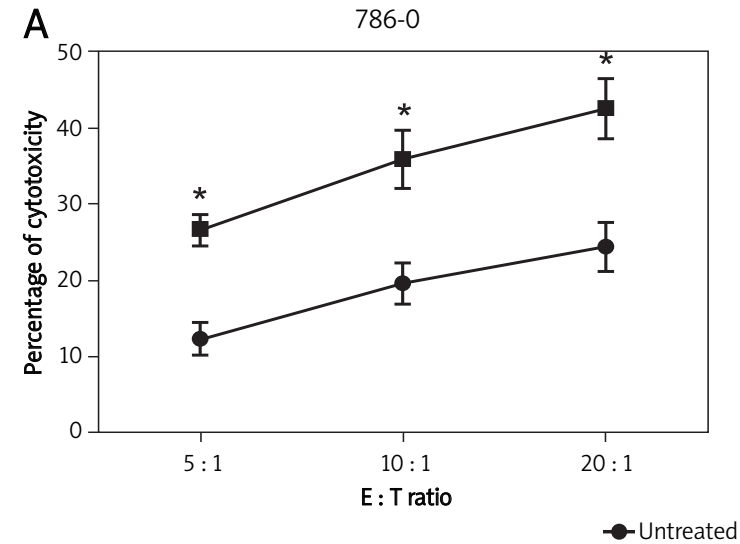

A activation of NK cells via NKG2D, we tested whether the renal carcinoma cells treated with VPA could lead to increase of NK cell degranulation. We analyzed the degranulation of NK-92 cells against 786-O and ACHN cells, by evaluating the expression of the lysosomal marker CD107a on the effector cells by FCM analysis correlated with NK cell cytotoxicity.

As shown in Figure 4, basal expression of CD107a in NK cells contacting 786-O or ACHN target cells was enhanced following treatment with $4 \mathrm{mM}$ VPA. The assay was performed at an E/T ratio of $2: 1$.

Combined with the above result that VPA treatment could upregulate the expression levels of NKG2D ligands, we demonstrated that augmented expression of NKG2D ligands in 786-O and ACHN cells enhanced NK cell degranulation by promoting recognition of tumor cells by NK cells.

\section{Discussion}

Renal carcinoma cells are resistant to conventional cytostatic agents, resulting in disappointing clinical outcomes. The response rates are very low, often just $5-15 \%$, and most responses are short lived [3]. However, recent studies found that renal
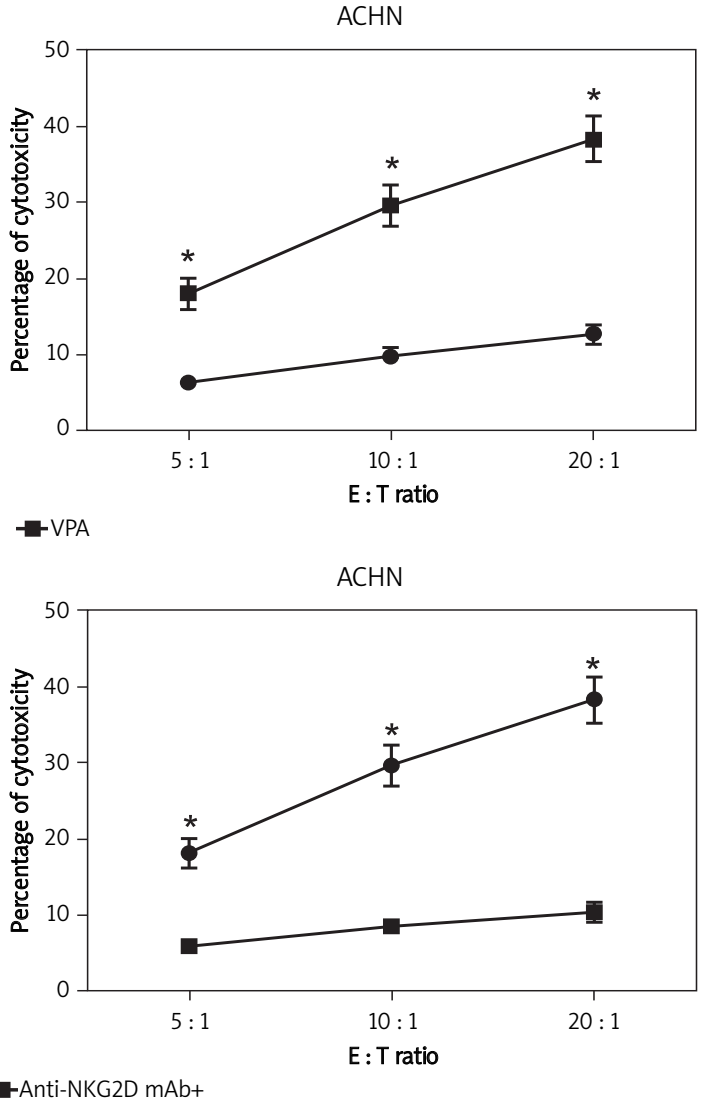

Figure 3. VPA enhances the susceptibility of renal carcinoma cells to NK cell-mediated cytotoxicity. A-786-O and ACHN cells were incubated with or without $4 \mathrm{mM}$ VPA for $24 \mathrm{~h}$, then the cytotoxicity of cultured NK cells against renal carcinoma cells was assessed; ${ }^{*} p<0.05$. B - 786-O and ACHN cells treated with 4 mM VPA were incubated in the presence or absence of anti-NKG2D mAb, then tested for their sensitivity to NK cell-mediated lysis; ${ }^{*} p<0.05$ Each bar indicates a mean $\pm S D$ of 3 samples. $E: T$ - effector : target 

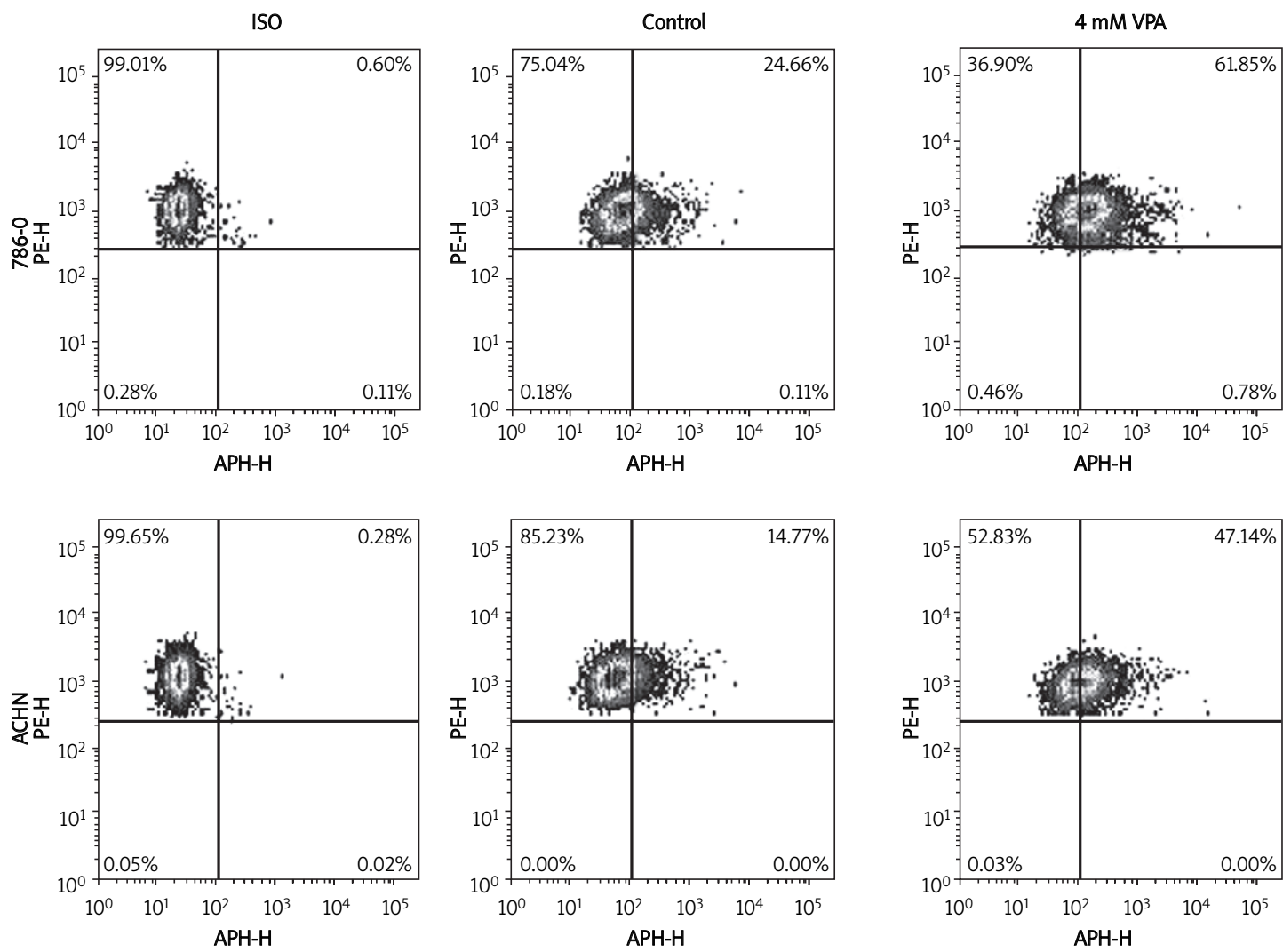

Figure 4. Influence of VPA on NK-92 cell degranulation against renal carcinoma cells. NK-92 cells were incubated with 786-O and ACHN cells, untreated or treated with $4 \mathrm{mM}$ VPA for $48 \mathrm{~h}$, and used as target cells in a degranulation assay. The assay was performed at an E/T ratio of $2: 1$. After $2 \mathrm{~h}$ at $37^{\circ} \mathrm{C}$, cells were stained with anti-CD56, anti-CD3 and anti-CD107a mAbs. Cell surface expression of CD107a was analyzed on CD56 ${ }^{+}$CD3 $^{-}$cells. Results are representative of one of three independent experiments

carcinoma cells can respond well to immunotherapy, especially metastatic renal carcinoma cells [20]. Valproic acid, a histone deacetylase inhibitor, has been found to display antineoplastic activity [21, 22]. However, the functional consequences of VPA treatment for cellular immunity in renal carcinoma cells remain unclear. In this study, we found that treatment of renal carcinoma cells with VPA upregulates the expression levels of NKG2DL, leading to significantly enhanced susceptibility of tumor cells to NK cell-mediated cytotoxicity.

In the last few years, increasing evidence has shown that anticancer immune responses may contribute to the control of tumors after conventional chemotherapy. A number of different observations have indicated that some chemotherapeutic agents can induce specific immune responses that result in immunogenic cancer cell death or immune stimulatory side effects [23, 24]. In this regard, tumor cells can be stressed by a variety of stimuli (intrinsic or extrinsic) that may induce membrane expression or release of specific signals such as danger signals, eat-me signals, or killing signals. These signals may facilitate immune recognition/activation and enhance the eradication of stressed/treated tumor cells [25]. There are various agents that can increase the expression of NKG2D ligands such as genotoxic agents, histone deacetylase inhibitors (e.g. VPA), or proteasome inhibitors (e.g. bortezomib), and facilitate the activation of NKG2Dexpressing lymphocytes (including NK cells, NK T lymphocytes, and cytotoxic $T$ lymphocytes) and tumor cell lysis [23, 24]. In this study, we focused on the influence of VPA because it has been shown to exhibit profound antitumor effects in a broad range of tumor entities $[12,13]$, and its low toxicity allows the use of other agents in combination.

By using FCM analysis, we investigated the effect of VPA on the expression of NKG2D ligands and $\mathrm{HLA}-\mathrm{A} / \mathrm{B} / \mathrm{C}$ ligands in human renal carcinoma cell lines. The effect of VPA on various cancer cells has been extensively studied, with inconsistent results. VPA increases the transcription of MICA/B without affecting ULBPs in hepatoma cells [12]. Poggi et al. found that VPA increases the expression level of MICA, ULBP-2, and ULBP-3 by acute myelocytic leukemia cells in vivo [26]. There were also studies showing upregulation of ULBP-1 and MICA/B on primary leukemic cells [27]. Lu et al. suggested that the expression of MICA/B and ULBP-2 was appar- 
ently upregulated after incubation with VPA. In our study, VPA upregulated the expression of most NKG2D ligands (MICA/B, ULBP-1, -2 in 786-O cell lines and MICA/B, ULBP1-3 in ACHN cell line) on the surface of two renal carcinoma cell lines. We suggested that this upregulation effect of VPA further results in its increased efficiency to activate NK cell degranulation as reported with other tumor cells [12, 13].

Previous studies have shown that agents which upregulated NKG2DLs induced apoptosis in various cells $[28,29]$ and experimental data might be influenced by the agents due to the apoptotic cells. In order to minimize the influence, we examined cell viability using a propidium iodide staining experiment and selected a concentration of $4 \mathrm{mM}$ for further analysis. A previous study used the same magnitude dosage of VPA (0.62-10.0 mM) to treat cultured thoracic cancer cells and suggested that VPA mediated significant cell line- and dose-dependent growth inhibition [30]. We chose $4 \mathrm{mM}$ of VPA in this study because this dosage did not significantly change the cell viability. Therefore, the dosage may not be of guiding significance to clinical dosing. The clinical dosing of VPA depends on which disease is being treated and whether VPA is being treated for maintenance or acute application.

HLA class I molecules suppress the activity of NK cells through inhibitory receptors [31]. In our study, the expression of HLA class I in renal carcinoma cells was not significantly changed in the presence of VPA. A similar effect was also observed in a previous study in human osteosarcoma cells [13]. In that study, VPA at 0.5 and $1.0 \mathrm{mM}$ did not increase HLA class I expression in MG-63 cells on days 3 and 7. However, in the other two cell lines of HOS cells and SaOS-2 cells, HLA class I was significantly increased on days 3 and 7 . This expression difference of HLA class I may result from the difference of cell lines. Our results suggested that, in renal carcinoma cells, VPA mainly increases the expression levels of cell-surface NKG2D ligands rather than HLA class I.

We next determined the effect of VPA on susceptibility of renal carcinoma cells to NK cell cytotoxicity using NK-92 cells. NK-92 was shown to exhibit substantial antitumor activity against melanoma and leukemia in vitro as well as in xenografted SCID mice [32]. In our study, the percentage of NK cell cytotoxicity was significantly increased following treatment of cells with VPA compared to untreated cells. This cytotoxicity was critically dependent on the interaction of NKG2D and its ligands, because the enhancing effect of VPA was blocked by the pretreatment of cells with anti-NKG2D mAbs. This result suggested that NKG2DL expression renders renal carcinoma cells susceptible to NK cell-mediated killing that can be markedly enhanced by VPA treatment.
NK cells are effector cells of the innate immune system and their functions are regulated by a number of killer cell-inhibitory and activating receptors. Depending on their intracellularly located immunoreceptor tyrosine-based inhibitory motif or activatory motifs, these receptors can mediate NK cell activation or tolerance toward self-Ags and/or tumor cells [33]. In this regard, evidence is accumulating that, apart from classical missing-self control or specific genotoxic-mediated activation of NKG2D ligands, different additional activating signals originating from a stressed tumor cell can contribute to NK cell recognition and cytotoxic action.

NKG2D is expressed in NK cells and other cytotoxic immune cells, such as CD8+ T cells and $\delta \gamma T$ cells. Therefore, VPA likely enhances the susceptibility of renal carcinoma cells to these cytotoxic immune cells. VPA has also been shown to enhance the susceptibility of tumor cells to Fas-ligandinduced cell death [34]. These results suggested that VPA in combination with immunotherapy activating cytotoxic immune cells could be useful to treat renal carcinoma.

In conclusion, our results indicated that VPA can clearly induce the expression of NKG2D ligands of renal carcinoma cell lines, thereby enhancing the cytotoxicity of NK cells against renal carcinoma cell lines. Our findings could provide evidence for immune-biologic therapy of kidney cancer - a new approach to cancer therapy.

\section{Acknowledgments}

Fengqiang Yang and Yang Shao should be regarded as co-first authors.

This work was partially supported by grants from the National Natural Science Foundation of China (No. 81270831, 81000311 and 81071856 ).

\section{References}

1. Thoenes W, Störkel S, Rumpelt H. Histopathology and classification of renal cell tumors (adenomas, oncocytomas and carcinomas): the basic cytological and histopathological elements and their use for diagnostics. Pathol Res Pract 1986; 181: 125-43.

2. Rini BI, Campbell SC, Escudier B. Renal cell carcinoma. Lancet 2009; 373: 1119-32.

3. Mulders PF, Brouwers AH, Hulsbergen-van der Kaa CA, van Lin EN, Osanto S, de Mulder PH. Guideline 'Renal cell carcinoma'. Ned Tijdschr Geneeskd 2008; 152: 376-80.

4. Moretta L, Moretta A. Unravelling natural killer cell function: triggering and inhibitory human NK receptors. EMBO J 2003; 23: 255-9.

5. Lanier LL. A renaissance for the tumor immunosurveillance hypothesis. Nat Med 2001; 7: 1178-80.

6. Raulet DH. Roles of the NKG2D immunoreceptor and its ligands. Nat Rev Immunol 2003; 3: 781-90.

7. Bauer S, Groh V, Wu J, et al. Activation of NK cells and T cells by NKG2D, a receptor for stress-inducible MICA. Science 1999; 285: 727-9. 
8. Groh V, Rhinehart R, Secrist H, Bauer S, Grabstein KH, Spies T. Broad tumor-associated expression and recognition by tumor-derived gammadelta T cells of MICA and MICB. Proc Natl Acad Sci USA 1999; 96: 6879-84.

9. Radosavljevic M, Cuillerier BI, Wilson MJ, Clément O, Wicker $\mathrm{S}$. A cluster of ten novel MHC class I related genes on human chromosome 6q24. 2-q25. 3. Genomics 2002; 79: $114-23$

10. Carayannopoulos LN, Naidenko OV, Fremont DH, Yokoyama WM. Cutting edge: murine UL16-binding proteinlike transcript 1: a newly described transcript encoding a high-affinity ligand for murine NKG2D. J Immunol 2002; 169: 4079-83.

11. Sakajiri S, Kumagai T, Kawamata N, Saitoh T, Said JW, Koeffler HP. Histone deacetylase inhibitors profoundly decrease proliferation of human lymphoid cancer cell lines. Exp Hematol 2005; 33: 53-61.

12. Armeanu S, Bitzer M, Lauer UM, et al. Natural killer cellmediated lysis of hepatoma cells via specific induction of NKG2D ligands by the histone deacetylase inhibitor sodium valproate. Cancer Res 2005; 65: 6321-9.

13. Yamanegi K, Yamane J, Kobayashi K, et al. Sodium valproate, a histone deacetylase inhibitor, augments the expression of cell-surface NKG2D ligands, MICA/B, without increasing their soluble forms to enhance susceptibility of human osteosarcoma cells to NK cell-mediated cytotoxicity. Oncol Rep 2010; 24: 1621-7.

14. Duenas-Gonzalez A, Candelaria M, Perez-Plascencia C, Perez-Cardenas E, De La Cruz-Hernandez E, Herrera LA. Valproic acid as epigenetic cancer drug: preclinical, clinical and transcriptional effects on solid tumors. Cancer Treat Rev 2008; 34: 206-22.

15. Thelen P, Schweyer S, Hemmerlein B, Wuttke W, Seseke F, Ringert $\mathrm{RH}$. Expressional changes after histone deacetylase inhibition by valproic acid in LNCaP human prostate cancer cells. Int J Oncol 2004; 24: 25-31.

16. Hummon AB, Lim SR, Difilippantonio MJ, Ried T. Isolation and solubilization of proteins after TRIzol® extraction of RNA and DNA from patient material following prolonged storage. Biotechniques 2007; 42: 467-70.

17. Heid CA, Stevens J, Livak KJ, Williams PM. Real time quantitative PCR. Genome Res 1996; 6: 986-94.

18. Lichtenfels R, Biddison WE, Schulz H, Vogt AB, Martin R. CARE-LASS (calcein-release-assay), an improved fluorescence-based test system to measure cytotoxic T lymphocyte activity. J Immunol Methods 1994; 172: 227-39.

19. Seize TK. Student's t-test. South Med J 1977; 70: 1299.

20. Guida M, Colucci G. Immunotherapy for metastatic renal cell carcinoma: is it a therapeutic option yet? Ann Oncol 2007; 18 Suppl 6: vi149-52.

21. Blaheta RA, Cinatl J Jr. Anti-tumor mechanisms of valproate: a novel role for an old drug. Med Res Rev 2002; 22: 492-511.

22. Gottlicher M, Minucci S, Zhu P, et al. Valproic acid defines a novel class of HDAC inhibitors inducing differentiation of transformed cells. EMBO J 2001; 20: 6969-78.

23. Ullrich E, Bonmort M, Mignot G, Kroemer G, Zitvogel L. Tumor stress, cell death and the ensuing immune response. Cell Death Differ 2007; 15: 21-8.

24. Zitvogel L, Apetoh L, Ghiringhelli F, Kroemer G. Immunological aspects of cancer chemotherapy. Nat Rev Immunol 2008; 8: 59-73.

25. Bryceson YT, March ME, Barber DF, Ljunggren HG, Long EO. Cytolytic granule polarization and degranulation controlled by different receptors in resting NK cells. J Exp Med 2005; 202: 1001-12.

26. Poggi A, Catellani S, Garuti A, Pierri I, Gobbi M, Zocchi MR. Effective in vivo induction of NKG2D ligands in acute myeloid leukaemias by all-trans-retinoic acid or sodium valproate. Leukemia 2009; 23: 641-8.

27. Diermayr S, Himmelreich H, Durovic B, et al. NKG2D ligand expression in $\mathrm{AML}$ increases in response to HDAC inhibitor valproic acid and contributes to allorecognition by NK-cell lines with single KIR-HLA class I specificities. Blood 2008; 111: 1428-36.

28. Kawagoe R, Kawagoe H, Sano K. Valproic acid induces apoptosis in human leukemia cells by stimulating both caspase-dependent and -independent apoptotic signaling pathways. Leuk Res 2002; 26: 495-502.

29. Kaiser M, Zavrski I, Sterz J, et al. The effects of the histone deacetylase inhibitor valproic acid on cell cycle, growth suppression and apoptosis in multiple myeloma. Haematologica 2006; 91: 248-51.

30. Yeow WS, Ziauddin MF, Maxhimer JB, et al. Potentiation of the anticancer effect of valproic acid, an antiepileptic agent with histone deacetylase inhibitory activity, by the kinase inhibitor Staurosporine or its clinically relevant analogue UCN-01. Br J Cancer 2006; 94: 1436-45.

31. Waldhauer I, Steinle A. NK cells and cancer immunosurveillance. Oncogene 2008; 27: 5932-43.

32. Tonn T, Becker S, Esser R, Schwabe D, Seifried E. Cellular immunotherapy of malignancies using the clonal natural killer cell line NK-92. J Hematother Stem Cell Res 2001; 10: 535-44.

33. Moretta L, Bottino C, Pende D, Castriconi R, Mingari MC, Moretta A. Surface NK receptors and their ligands on tumor cells. Elsevier 2006

34. Yamanegi K, Yamane J, Hata M, et al. Sodium valproate, a histone deacetylase inhibitor, decreases the secretion of soluble Fas by human osteosarcoma cells and increases their sensitivity to Fas-mediated cell death. J Cancer Res Clin Oncol 2009; 135: 879-89. 